\title{
The crural interosseous membrane re-visited: a histological and microscopic study
}

\author{
Joseph Morley (1), Chenglei Fan (2), Kena McDermott (1), Caterina Fede (2), Emmett \\ Hughes (1), Carla Stecco (2)
}

(1) University of Bridgeport School of Chiropractic, Bridgeport, CT, USA; (2) Department of Neurosciences, Institute of Human Anatomy, University of Padova, Padova, Italy

This article is distributed under the terms of the Creative Commons Attribution Noncommercial License (CC BY-NC 4.0) which permits any noncommercial use, distribution, and reproduction in any medium, provided the original author(s) and source are credited.

\begin{abstract}
The aim of this study was to characterize the microscopic structure and sensory nerve endings of the crural interosseous membrane (IM). 13 IMs from 7 cadavers were used to analyze the organization of the collagen fibers, IM's thickness, distribution of elastic fibers and nerve elements. The IM is mainly a two-layer collagen fascicle structure with the collagen fibers of adjacent layers orientated along different directions, forming angles of $30.5+/-1.7^{\circ}$ at proximal and $26.6+/-2.1^{\circ}$ at distal part $(\mathrm{P}>0.05)$. The percentage of elastic fibers between the two layers and inside the collagen fascicle layer is $10.1+/-0.5 \%$ and $2.2+/-0.1 \%(\mathrm{P}<0.001)$. The IM's thickness at proximal, middle, and distal parts is $268.5+/-18.6 \mu \mathrm{m} ; 293.2+/-12.5 \mu \mathrm{m} ; 365.3+/$ $19.3 \mu \mathrm{m}$, respectively (Proximal vs Distal: $\mathrm{P}<0.001$; Middle vs Distal: $\mathrm{P}<0.05$ ). Nerve elements were present and located both inside and on the surface of the IM, whereas the mechanoreceptors are mainly located on the surface of the IM. Free nerve endings $\left(33.3+/-5.0 / \mathrm{cm}^{2}\right)$ and Ruffini corpuscles $\left(3.4+/-0.6 / \mathrm{cm}^{2}\right)$ were the predominant sensory elements, while Pacinian corpuscles $\left(1.3+/-0.7 / \mathrm{cm}^{2}\right)$ were rarely found. The type of mechanoreceptors found suggests that the IM may play a role in proprioception.
\end{abstract}

Key Words: crural interosseous membrane; sensory nerve ending; mechanoreceptor.

Eur J Transl Myol 29 (3): 164-170, 2019

The crural interosseous membrane (IM) extends between the interosseous crests of the tibia and fibula, helps stabilize the tibio-fibular relationship and separates anterior compartment muscles from posterior compartment muscles of the leg. Previous microscopic and anatomical studies of the crural IM have shown the angulation of fibers, ${ }^{1,2}$ diameter of IM fiber bundles, ${ }^{3}$ stress and strain characteristics, ${ }^{4}$ and dimensions of the IM. $^{5}$ The IM is comprised of a main fibrous network of bundles of large diameter fibers and a secondary network of fine fibers interspersed between the main network. ${ }^{4}$ The fibers comprising the IM have been studied for their ability to withstand stretching both parallel and perpendicular to the direction of the fibers. Minns and Hunter assessed the stress/strain characteristics of the IM. ${ }^{4}$ They showed that the IM was able to be stretched an additional $7.7 \%(+2.1)$ of its initial length and $40.5 \%$ $(+7.1)$ of its initial width before rupturing. The tensile stress required to rupture the fibers was $920(+205)$ $\mathrm{kg} / \mathrm{cm}^{2}$ parallel to the direction of fibers and $41(+13)$ $\mathrm{kg} / \mathrm{cm}^{2}$ perpendicular to the direction of the fibers. These studies suggested that IM seems have a certain adaptability, but up to now nobody knows if this adaptability is due to the elastic component or to the collagen conformation. In addition, shin splints are defined as pain in the medial or postero-medial lower leg, brought on by running, walking or other activity involving the lower leg. Although the IM has been suggested as a possible cause of shin splints by O’Donaghue, ${ }^{6}$ no definitive research has been done to confirm this theory. ${ }^{7}$ The fact that several muscles involved in gait have their origins on the IM provided the impetus for investigating whether the IM might have nerve receptors influencing gait. To the best of our knowledge, no studies about the innervation of the crural IM are present in the literatures, a Pubmed search was done, combining the term interosseous membrane with mechanoreceptors, Pacinian corpuscles, Ruffini corpuscles, or nociceptors. The main purpose of our study was to investigate the microscopic anatomy and the innervation, if any, of the IM. We hope that this evaluation will lead to a better understanding of the IM's role in pain, proprioception, motor coordination and tibio-fibular stabilization during gait, and if a specific 


\section{Crural interosseous membrane re-visited}

Eur J Transl Myol 29 (3): 164-170, 2019

conformation of the collagen and elastic fibers could be recognized.

\section{Materials and Methods}

All dissections were done at the University of Bridgeport, USA. Permission to dissect cadavers was obtained from the Director of the cadaver laboratory at the University of Bridgeport, USA. The IMs harvested were dissected from embalmed human cadavers that were purchased by University of Bridgeport for the purposes of scientific research and were exempt from IRB protocol review. Fourteen IMs were dissected from seven bodies of both female and male identities of mixed adult ages. The skin, adipose tissue and muscles attached to both the anterior and posterior aspects of the membranes were removed, while some muscle fibers still remain. The IMs were cut directly against the tibia and fibula in a proximal to distal direction, releasing the IM from the bone. Great care was taken to ensure the entire IM was obtained from each leg. Thirteen specimens harvested were deemed adequate for further processing. One IM was found to be too dry to be usable for further processing, due to previous dissection of surrounding tissues. After the IMs were removed, the length, width and depth of specimens were measured, recorded and labeled according to cadaver and right or left limb (Table1). The ends of each IM were then inked to identify proximal and distal ends of the specimens. Specimens were then cut in half, labelled proximal or distal and mounted over gauze on cardboard and immediately post-fixed in $10 \%$ neutral buffered formalin (10\% NBF), pH 7.4 at $4{ }^{\circ} \mathrm{C}$. The 26 samples were then air transported to the University of Padova, Italy for further histological processing. Prior to histological analysis of the IMs, the organization of the main collagen fascicles were evaluated with a light that permitted transillumination of the IM (a light was placed behind the IM). In order to evaluate any differences in the innervations of the IM, three representative samples, measuring $1.5 \mathrm{~cm}$ length $\mathrm{x} 1.0 \mathrm{~cm}$ width, were taken from the proximal, medial and distal portions of each IM specimen. All representative samples were then submitted for processing prior to paraffin embedding.

Sections of $5 \mu \mathrm{m}$ were cut and treated with a solution of $\mathrm{H}_{2} \mathrm{O}_{2} 0.5 \%$ for 15 minutes in order to inhibit endogenous peroxidase activity, immediately followed by 3 washings in phosphate buffered saline (PBS). Slides were then incubated with bovine serum albumin (BSA) $0.2 \%$ for 1 hour before being treated with primary antibody S100, 1:4000 (Polyclonal Rabbit Anti-S100, Dako, Denmark) in BSA at $4^{\circ} \mathrm{C}$ overnight. After 3 washings in PBS, the sections were incubated with the secondary antibody (Goat anti-rabbit IgG peroxidase-coniugated antibodies DAKO) 1:300 for 1 hour and, after repeated washings, the reaction was developed with 3,3'-diaminobenzidine (Liquid DAB Substrate Chromogen System; Dako). The negative controls were obtained by omitting the primary antibody. Finally, each slide was counterstained with hematoxylin, followed by dehydration in a graded ethanol series, and mounted for microscopic evaluation using a Leica DMR microscope. In addition, sections from all samples were stained with Hematoxylin and eosin stain (H\&E), Verhoeff Van Gieson (VVG) and Azan Mallory Trichrome (AM) stain in order to highlight

Table 1. Measurements performed on the crural interosseous membranes after dissection (cm) $($ Mean +/- SEM; $N=13)$

\begin{tabular}{|c|c|c|c|c|}
\hline Cadaver ID & & ength & Proximal Width & Distal Width \\
\hline \multirow[t]{2}{*}{ A } & $\mathrm{R}$ & 25.5 & 3.0 & 0.9 \\
\hline & $\mathrm{L}$ & 27.0 & 2.5 & 0.6 \\
\hline \multirow[t]{2}{*}{ B } & $\mathrm{R}$ & 29.5 & 3.0 & 0.7 \\
\hline & $\mathrm{L}$ & 29.4 & 2.7 & 0.7 \\
\hline \multirow[t]{2}{*}{$\mathrm{C}$} & $\mathrm{R}$ & 30.0 & 2.5 & 0.5 \\
\hline & $\mathrm{L}$ & 29.0 & 2.5 & 0.5 \\
\hline \multirow[t]{2}{*}{$\mathrm{D}$} & $\mathrm{R}$ & 25.0 & 2.3 & 0.5 \\
\hline & $\mathrm{L}$ & 29.0 & 2.3 & 0.5 \\
\hline \multirow[t]{2}{*}{$\mathrm{E}$} & $\mathrm{R}$ & 29.0 & 3.0 & 0.8 \\
\hline & L-NO & - & - & - \\
\hline \multirow[t]{2}{*}{$\mathrm{F}$} & $\mathrm{R}$ & 29.0 & 2.5 & 0.7 \\
\hline & $\mathrm{L}$ & 26.0 & 2.5 & 0.4 \\
\hline \multirow[t]{2}{*}{ G } & $\mathrm{R}$ & 27.5 & 2.7 & 0.9 \\
\hline & $\mathrm{L}$ & 29.5 & 2.0 & 0.7 \\
\hline Mean +/- SEM & & $28.1+/-0.5$ & $2.5+/-0.1$ & $0.7+/-0.1$ \\
\hline
\end{tabular}

Note. L-NO: one IM was found to be too dry to be usable for further processing, due to previous dissection of surrounding tissues. 


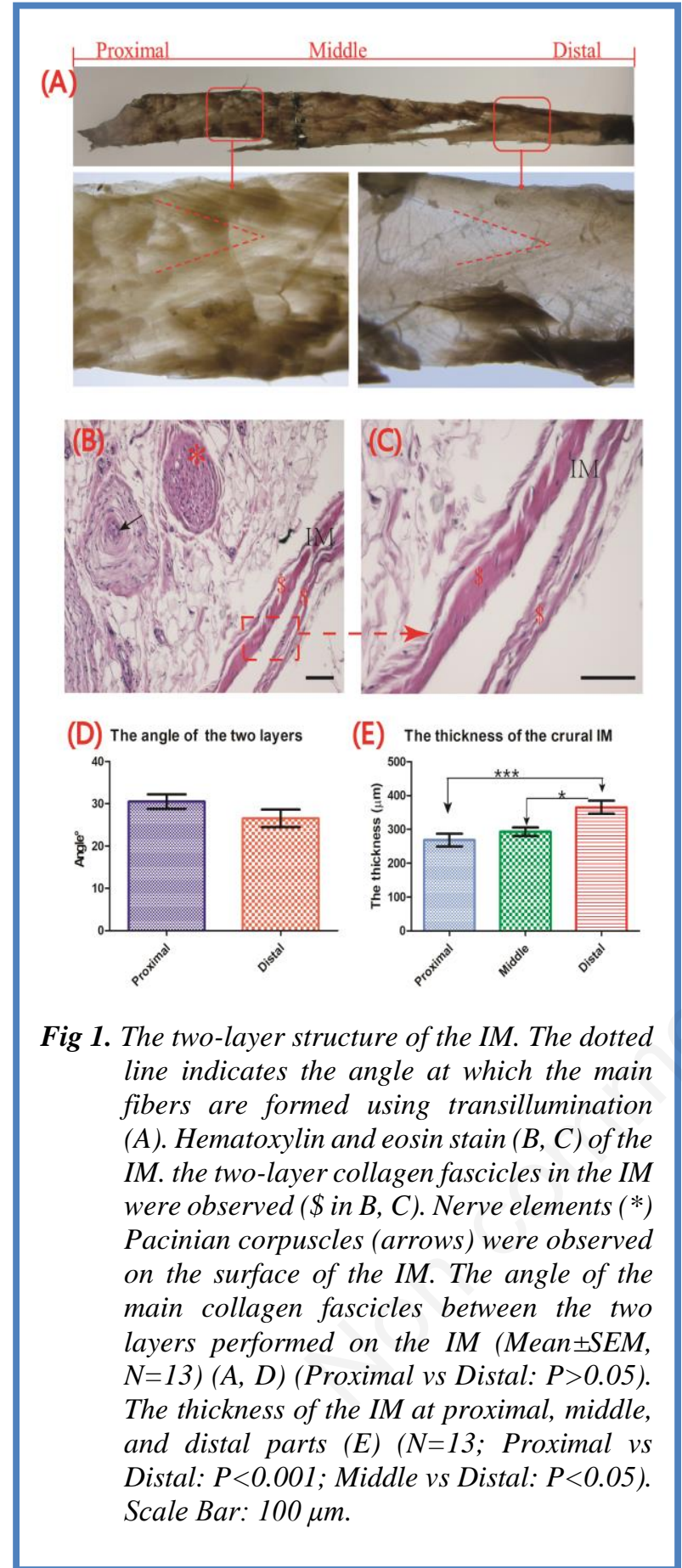

tissue morphology. VVG is used to stain the elastic fibers (black-violet color), while AM is used to stain the collagen fibers (blue). Histologic evaluation of the vessels, elastin and collagen fiber arrangements in the specimens was conducted using an optical microscope (Leica DMR). The mechanoreceptors were comparatively observed and manually counted in serial sections stained with immunohistochemical methods at an enlargement of $20 \times$ or $40 \times$. The diameters of the mechanoreceptors and the thickness of the IM were measured with ImageJ software.

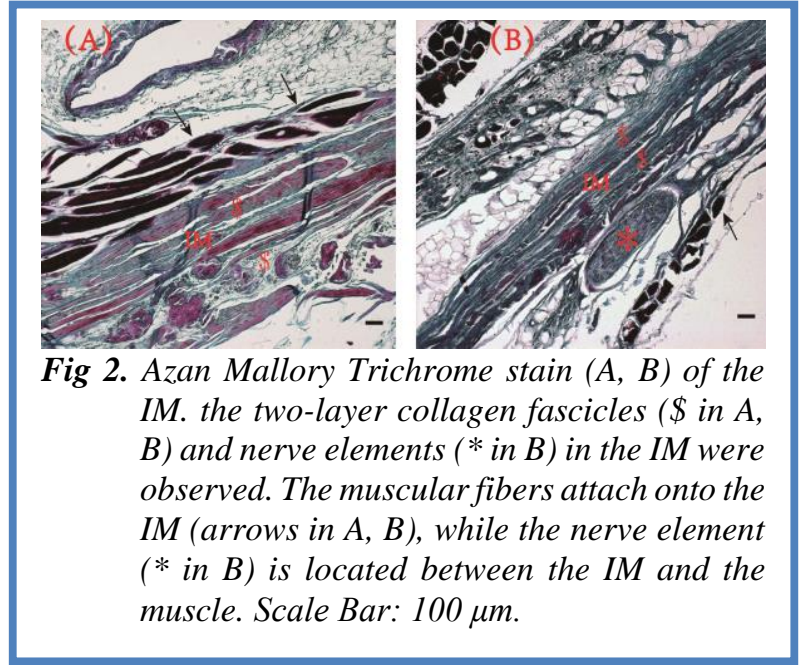

\section{Statistical analysis}

Results are expressed as mean and standard error of mean (SEM) of values obtained from 13 IMs; Graph Pad Prism 6 (GraphPad Software Inc., San Diego, CA) was used to test for statistically significant differences. The values represent the number of sensory nerve endings per $\mathrm{cm}^{2}$. All groups of sensory nerve endings were found to not have normal distributions. To analyse the quantity different type of sensory nerve endings within the specimens (3 tests, 3 sensory nerve endings ) and the proximal, middle, distal distribution densities of sensory nerve endings of the crural IM ( 3 tests, 3 parts: proximal, middle, distal ), the subsequent statistical analysis was performed using the Kruskal-Wallis test followed by the Mann-Whitney test with post hoc Bonferroni adjustment. To analyse the proximal, middle, distal thickness of the crural IM, the proximal, distal angle of the crural IM, elastic fiber percentage, as all of the data were normally distributed, the proximal, middle, distal thickness of the crural IM, groups were compared using One-way ANOVA with Bonferroni's Multiple Comparison Test. The level of significance was considered high with $\mathrm{P} \leq 0.05$. The proximal, distal angle of the crural IM, elastic fiber percentage was conducted using a t-test. The level of significance was considered high with $\mathrm{P} \leq 0.05$.

\section{Results}

Macroscopic analysis of the dissected crural IMs reveals mainly a two-layer structure comprised of collagen fibers with muscular fibers attaching to the membrane. The mean length of the IMs measured is $28.1+/-0.5 \mathrm{~cm}$. In the frontal plane, the proximal part is larger, with a mean width of $2.5+/-0.1 \mathrm{~cm}$, whilst distally the mean width is $0.7+/-0.1 \mathrm{~cm}(\mathrm{~N}=13$; Table 1$)$. It gives rise to attachment of many muscular fibers anteriorly and posteriorly, predominantly in the proximal segment. The collagen fiber bundles aligned mainly along the longitudinal and oblique directions and are less compact in the transverse direction. The collagen fibers within each layer are 


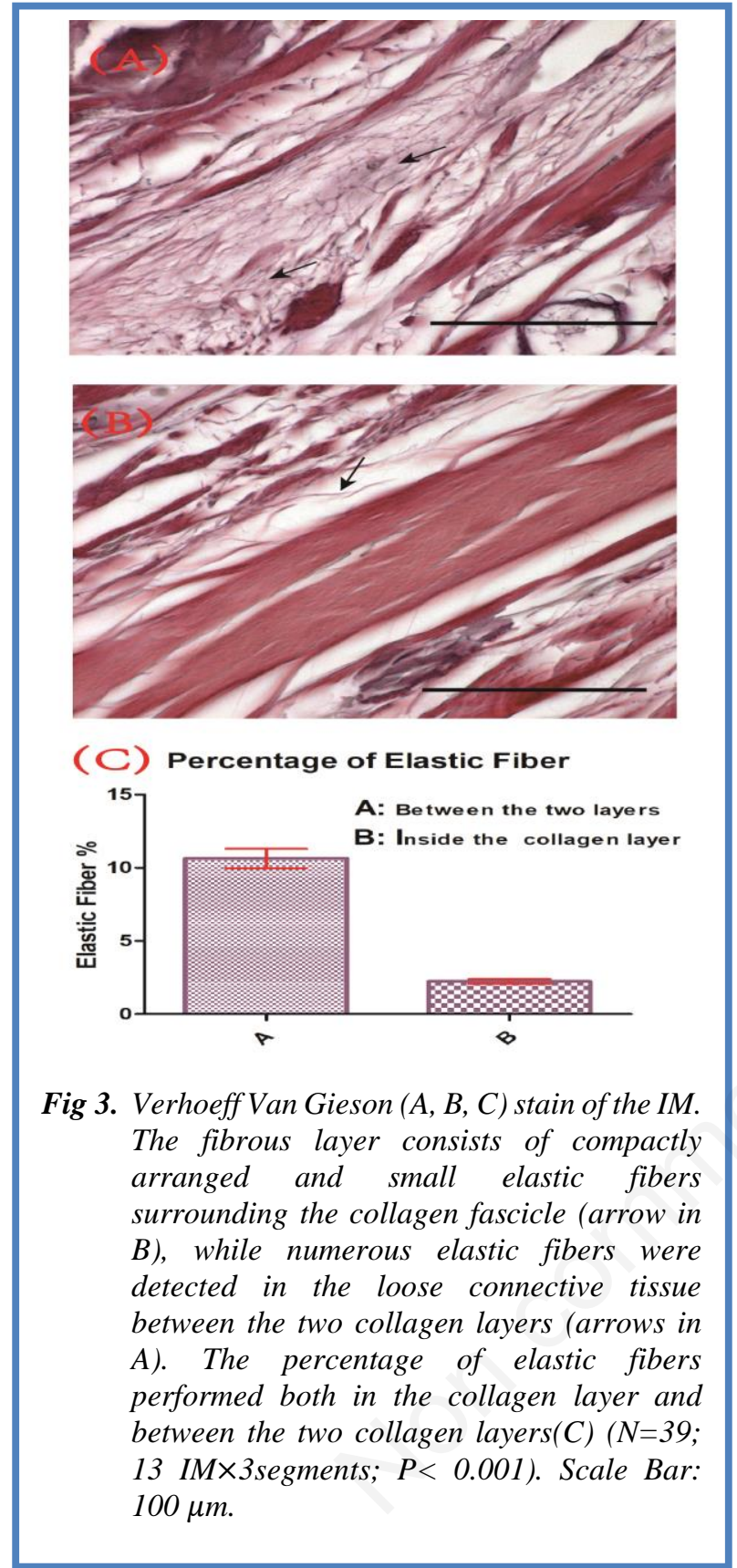

oriented in the same direction and ran oblique distally, while the collagen fibers of adjacent layers are orientated along different directions that form angles. The angle of the main collagen fascicles between the two layers is 30.5 $+/-1.7^{\circ}$ in the proximal part and $26.6+/-2.1^{\circ}$ in the distal part using transillumination. There is no statistically significant difference between the proximal and distal part $(\mathrm{N}=13 ; \mathrm{P}>0.05)$ (Figure.1 a, d). The mean thicknesses of the proximal, middle and distal parts of the IM are $268.5+/-18.6 \mu \mathrm{m} ; 293.2+/-12.5 \mu \mathrm{m} ; 365.3+/$ $19.3 \mu \mathrm{m}$, respectively $(\mathrm{N}=13$; Proximal vs Distal: $\mathrm{P}<0.001$, distal greater; Middle vs Distal: $\mathrm{P}<0.05$, distal greater). Statistically significant differences were found between the proximal and distal thicknesses and the

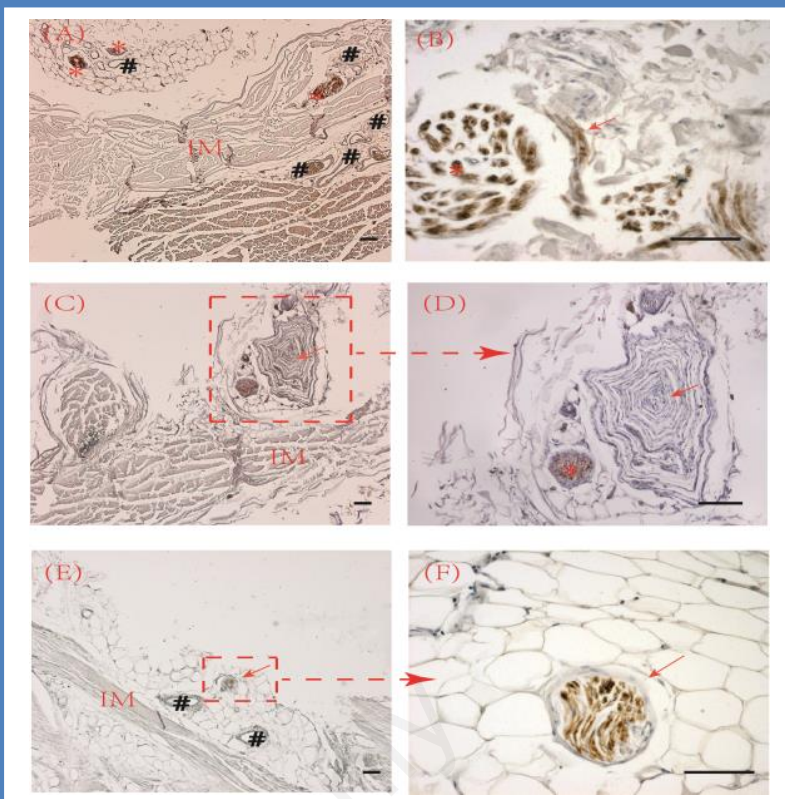

Fig 4. Crural IM stained with S100. The IM is visible in the overview magnification $(A, C$, $E)$ with blood vessels (\# in $A, E$ ) and nerve elements (*) inside and on the surface of the IM. Free nerve endings are mainly found on the surface of the IM, showing immunoreactivity with $S 100$ (arrow in B). Pacinian corpuscles (arrow in C, D), Ruffini corpuscles (arrow in E, F), are only located on the surface of the IM.

Scale Bar: $100 \mu \mathrm{m}$.

middle and distal thicknesses (Figure.1 e). Blood vessels and nerve elements were observed between the two layer and on the surface of the IM (Figure. 2 a, b; Figure. 4 a). The elastic fibers were found to be almost absent in the collagen layers and present mostly in the loose connective tissue between the two collagen layers (Figure. $3 \mathrm{a}, \mathrm{b}$ ), The percentage of elastic fibers between the two layers is $10.6+/-0.5 \%$, while inside the collagen fiber layer it is $2.2+/-0.1 \%(\mathrm{~N}=39 ; 13 \mathrm{IM} \times 3$ segments; $\mathrm{P}<0.001$ ) (Figure. $3 \mathrm{c}$ ).

\section{Observations of Immunohistochemical Labeling}

Nerve elements were present and located both inside (between the two layers) and on the surface of the IM in all the specimens analyzed; although the distribution of mechanoreceptors demonstrated that differences existed according to the area, or the specimens (Figure.4a). However, no nerve elements were detected inside the collagen layer in any of the sections. Free nerve endings were found in all $13 \mathrm{IMs}$, whereas Ruffini and Pacinian corpuscles were present only in some. Free nerve endings were mainly located on the surface of the IMs, while a few were located inside (between the two layers). Ruffini and Pacinian corpuscles were located only on the surface 
Crural interosseous membrane re-visited

Eur J Transl Myol 29 (3): 164-170, 2019

Table 2 The distribution densities and percentages of different types of mechano-receptors identified in 13 interosseous membranes (Mean \pm SEM; N=39; 13 IMx3segments)

\begin{tabular}{lllllll}
\hline Type & $\begin{array}{l}\text { Mean } \pm \text { SEM } \\
\left(\mathrm{cm}^{2}\right)\end{array}$ & Segments & $\begin{array}{l}\text { Mean } \pm \\
\text { SEM }\left(\mathrm{cm}^{2}\right)\end{array}$ & $\mathrm{Nu}$ & $\%$ & Morphology ${ }^{24,25}$ \\
\hline Ruffini & $3.4 \pm 0.6^{\#}$ & $\mathrm{P}$ & $4.3 \pm 0.3$ & 11 & 84.6 & $\begin{array}{l}\text { Clusters of three to six } \\
\text { ramifications }\end{array}$ \\
corpuscles & & $\mathrm{M}$ & $2.3 \pm 0.4$ & & & \\
Pacinian & $1.3 \pm 0.7$ & $\mathrm{D}$ & $3.4 \pm 0.3$ & & & \\
corpuscles & & $\mathrm{P}$ & $0.9 \pm 0.3$ & 5 & 38.5 & $\begin{array}{l}\text { Encapsulated in clusters } \\
\text { of two to four with an } \\
\text { ovoid or cylindrical } \\
\text { shape }\end{array}$ \\
$\begin{array}{llllll}\text { Free nerve } \\
\text { endings }\end{array}$ & $33.3 \pm 5.0^{* *}$ & $\mathrm{P}$ & $0.8 \pm 0.2$ & & & \\
& & $\mathrm{M}$ & $2.2 \pm 0.5$ & & & $\begin{array}{l}\text { Long and fine without a } \\
\text { define shape }\end{array}$ \\
\hline
\end{tabular}

Note: Nu: The number of IM positive for the mechanoreceptors. \%: Percentages of different types of mechanoreceptors identified in total of 13 interosseous membranes. P: Proximal; M: Middle; D: Distal. ** Significantly different compared to the other two corpuscles (Ruffini and Pacinian corpuscles), $P<0.001$. " Significantly different compared to Pacinian corpuscles, $P=0.0044$

of the IM where muscle fibers attach onto the membranes. No Ruffini or Pacinian corpuscles were found inside the IM (Figure 4c, d, e, f). Free nerve endings $\left(33.3+/-5.0 / \mathrm{cm}^{2}\right)$ were the predominant mechanoreceptor and were more common than all other types of sensory nerve endings ( $\mathrm{P}<0.001$, respectively) followed by Ruffini corpuscles $3.4+/-0.6 / \mathrm{cm}^{2}$ and Pacinian corpuscles $1.3+/-0.7 / \mathrm{cm}^{2}(\mathrm{~N}=39 ; 13 \mathrm{IM} \times$ 3 segments) (Table 2). The diameter sizes of the Ruffini and Pacinian corpuscles ranged between 30.7 and 258.2 $\mu \mathrm{m}$ and between 188.5 and $675.0+/-\mathrm{m}$, respectively. After reviewing the total sections in each sample, we found that Ruffini corpuscles are in 11 IMs, Pacinian corpuscles in 5 IMs and Free nerve endings in all 13 IMs. The structures of mechanoreceptors (free nerve endings, Ruffini and Pacinian corpuscles) were evaluated according to the method Freeman and Wyke. ${ }^{8}$ Under light microscope, a total of 16 Ruffini corpuscles ( 7 in the proximal segment; 3 in the middle segment; 6 in the distal segment) and 6 Pacinian corpuscles ( 2 in the proximal segment; 1 in the middle segment; 3 in the distal segment) were detected in all of the sections, while Free nerve endings were commonly present (Figure 4b). However, there was no significant difference between the proximal, middle and distal segments in the sensory nerve endings (Ruffini, Pacinian corpuscles and Free nerve ending) ( $\mathrm{N}=13 ; \mathrm{P}>0.1$ respectively).

\section{Discussion}

Anatomical and biomechanical considerations of the IM In this study, we found that the IM is mainly a two-layer collagen fascicle structure. The collagen fibers of the two adjacent layers are orientated along different directions, forming angles of $30.5+/-1.7^{\circ}$ at the proximal part and $26.6+/-2.1^{\circ}$ at the distal part. These results confirm the data reported by Minns and Hunter, ${ }^{4}$ who found that the fibers of the IM are angled at between $20^{\circ}$ and $30^{\circ}$ towards vertical and run distally from the tibia towards the fibula. Similarly, Norkus et al., ${ }^{2}$ stated that the IM fiber orientation is at $15^{\circ}-20^{\circ}$ towards vertical, and runs distally from tibia to the fibula. Elamrani et al. reported that fibers of the anterior layer made an angle of $13^{\circ}$ (SD 2.6) with the axis of fibula. ${ }^{5}$ Those of the posterior layer made an angle of $24.2^{\circ}$ (SD 2.48) with the axis of fibula. However, those researchers didn't measure the angle of the two adjacent collagen layers. The dimensions after harvesting is smaller than the dimension in situ according to Elamrani et al.; however the thickness of the IM is different than the data reported by Elamrani et al. ${ }^{5}$ They used a manual caliper while we measured the thickness of the microscopic image using ImageJ software. The elastic fibers were found to be almost absent in the collagen layers and present mostly in the loose connective tissue between the two collagen layers. This organization of these layers strongly resembles the microscopic structure of the aponeurotic fasciae, ${ }^{9-11}$ and suggests a similar mechanical function. That histological arrangement may be explained by the fact that there is more gliding between the two collagen layers than the collagen fiber layer due to the presence of loose connective tissue. The collagen layer of the IM may allow force transmission along longitudinal and oblique directions between the tibia and fibula, while the existence of elastic fibers and loose connective tissue between the two collagen layers may allow the 
adaptability of the IM in a transverse direction. Our results showed that the distal IM was thicker than the proximal and middle IM segments. This may correspond to the fact that the distal IM and the anterior and posterior tibiofibular ligaments are important for stabilizing the ankle joint. ${ }^{5,12}$ This histological finding also coincides with the fact that it is the distal tibio-fibular articulation that separates slightly when the runner's foot strikes the ground. ${ }^{2,13-19}$

\section{Nerve receptors of the crural IM}

Mechanoreceptors are responsible for the sensations of stretch, pressure, touch and vibration. They include Pacinian corpuscles, which are found in the skin, joint capsules and periosteum and adapt rapidly to stimuli. Ruffini corpuscles are found in the skin and joint capsules and adapt slowly to stimuli. ${ }^{20,21}$ So stretching, twisting or pressure affecting a joint, can activate Pacinian or Ruffini afferent corpuscles and affect efferent activity of relevant muscles. Interestingly, Adams et al. reports that free nerve endings, although categorized as non-specialized, can function as mechanoreceptors, nociceptors and thermoreceptors or in a polymodal manner. ${ }^{22}$ Recent studies have reported that fasciae present confirmatory innervation evidence. In the present study, free nerve endings were the most numerous nerve receptors found in the crural IMs, whilst, Pacinian and Ruffini corpuscles were located on the outside surface, where muscles originate on the IM. Based on these facts, free nerve endings and Ruffini receptors are present on the gliding surface and they sense the change of tension and the gliding between the IM, and the muscle that originates on the IM, during gait. Pacinian corpuscles are pressure receptors located in the loose connective tissue between the IM and the muscles that originate on the IM. In this way, it can sense the mechanical pressure of varying strength and frequency between the IM and the muscle during gait. The tibio-fibular joint is considered a syndesmodic joint, whilst, part of the tibio-fibular joint complex includes the IM. ${ }^{23-25}$ When the anterior compartment muscles contract, it will stretch the IM and increase the afferent signal of the mechanoreceptor to the central nervous system and then regulate the activation of the IM and/or posterior compartment muscles. This balances the tension of the IM, permitting its activation to be synchronized during dorsi and plantar flexion of the ankle joint. There are several theoretical explanations for only a few Pacinian corpuscles in our IM samples. First, the fact that our Pacinian corpuscles were found between the IM and muscle attachments to the IM are significant. Removal of the muscle fiber away from the IM could very easily damage or destroy any type of nerve receptors that would normally be present. Second, the margins of the IM, where it attaches to the tibia and fibula, are where mechanical stress is going to be greatest during walking or running. Cutting the IM away from the tibia and fibula may have damaged any Pacinian corpuscles present between the IM and tibia and fibula. The paucity of
Ruffini corpuscles detected in our IM samples is explained by the same reasoning as for the Pacinian corpuscles. Therefore, any future research in this area should consider doing dissections in such a way as to preserve any possible Pacinian or Ruffini corpuscles near attachments to bone and muscle. In conclusion, the type of mechanoreceptors found suggests that the IM can play a role in proprioception. The free nerve endings and Ruffini corpuscles mentioned above may perceive changes in IM tension, probably due to the action of the various muscular origins on the anterior and posterior compartments of the IM. The Pacinian corpuscles on the surface of the IM may sense any pressure changes that occur during gait. Hopefully, future researchers investigating the crural IM will evaluate the percentages of mechanoreceptors found at IM with muscle fibers still attached and be able to augment our findings. In addition, future studies are necessary to better illustrate the biomechanical properties of the IM under dynamic loading conditions. The authors of this article hope that they will be able to utilize the new findings from this study and apply them to the role of the crural IM in human gait.

\section{List of acronyms}

IM - interosseous membrane PBS-phosphate buffered saline

BSA-bovine serum albumin

H\&E-Hematoxylin and eosin stain

VVG-Verhoeff Van Gieson

AM-Azan Mallory Trichrome

SEM-standard error of mean

\section{Authors contributions}

$\mathrm{JM}, \mathrm{CF}, \mathrm{KM}, \mathrm{CF}, \mathrm{EH}, \mathrm{CS}$ equally participated in experimental design, data collection and analyses, manuscript writing and reviewing.

\section{Acknowledgments}

The authors would like to thank Kevin R. Kelliher, PhD, the director of the anatomy laboratory at the University of Bridgeport, for his help in collecting our biological specimens. The authors would also like to thank Stephen M. Perle, DC, MS, Professor of Clinical Sciences at the University of Bridgeport School of Chiropractic, for his help in facilitating the initial meeting for this study. The authors would also like to thank Dr. Lucia Petrelli and Dr. Damiana Incendi, Department of Neurosciences, Institute of Human Anatomy, University of Padova, for technical assistance. Funding None

\section{Conflict of Interest}

The authors declare they have no financial, personal, or other conflicts of interest.

\section{Ethical Publication Statement}

We confirm that we have read the Journal's position on issues involved in ethical publication and affirm that this report is consistent with those guidelines. 


\section{Crural interosseous membrane re-visited}

Eur J Transl Myol 29 (3): 164-170, 2019

\section{Corresponding Author}

Prof. Carla Stecco, Section of Anatomy, Department of Neuroscience, University of Padova, via A. Gabelli 65, 35121 Padova, Italy. Tel. +39.049.8272315 - Fax: +39.049 .8272328 .

E-mail: carla.stecco@unipd.it

E-mails of co-authors

Joseph Morley:jmorley11@hotmail.com

Chenglei Fan: chenglei.fan@studenti.unipd.it

Kena McDermott: mcdermottkena@gmail.com

Caterina Fede: caterina.fede@unipd.it

Emmett Hughes: ehughes@bridgeport.edu

\section{References}

1. Goss CM. Gray's anatomy. Lea and Febiger, Philadelphia; 1973.

2. Norkus SA, Floyd R. The anatomy and mechanisms of syndesmotic ankle sprains. J Athl Training 2001;36:68.

3. Murali S, Aspden R, Hutchison J, et al. Collagen organization in the crural interosseous membrane and its relationship to fibular osteotomy. Injury 1994;25:247-9.

4. Minns R, Hunter J. The mechanical and structural characteristics of the tibio-fibular interosseous membrane. Acta Orthop Scand 1976;47:236-40.

5. Elamrani D, Aumar A, Wavreille G, et al. Comparative morphometry of the antebrachial and crural interosseous membranes: preliminary study for the use of the crural interosseous membrane in the surgical repair of the antebrachial interosseous membrane tears. Surg Radiol Anat 2014;36:333-9.

6. O'Donoghue DH. Treatment of injuries to athletes: WB Saunders Company; 1984.

7. Kues J. Literature Review: The Pathology of Shin Splints. J Orthop Sport Phys 1990;12:115-21.

8. Freeman M, Wyke B. The innervation of the knee joint. An anatomical and histological study in the cat. J Anat 1967;101:505.

9. Stecco C, Corradin M, Macchi V, et al. Plantar fascia anatomy and its relationship with A chilles tendon and paratenon. J Anat 2013;223:665-76.

10. Stecco C, Gagey O, Belloni A, et al. Anatomy of the deep fascia of the upper limb. Second part: study of innervation. Morphologie 2007;91:38-43.

11. Stecco C, Macchi V, Porzionato A, et al. The ankle retinacula: morphological evidence of the proprioceptive role of the fascial system. Cells Tissues Organs 2010;192:200-10.

12. Ebraheim NA, Taser F, Shafiq Q, et al. Anatomical evaluation and clinical importance of the tibiofibular syndesmosis ligaments. Surg Radiol Anat 2006;28:142-9.

13. Anderson MK, Hall SJ. Sports injury management: Williams \& Wilkins; 1995.

14. Arndt A, Wolf P, Liu A, et al. Intrinsic foot kinematics measured in vivo during the stance phase of slow running. J Biomech 2007;40:2672-8.

15. Brosky T, Nyland J, Nitz A, et al. The ankle ligaments: consideration of syndesmotic injury and implications for rehabilitation. J Orthop Sport Phys 1995;21:197-205.

16. Katznelson A, Lin E, Militiano J. Ruptures of the ligaments about the tibio-fibular syndesmosis. Injury 1983;15:170-2.

17. Stiehl JB. Complex ankle fracture dislocations with syndesmotic diastasis. Orthopaedic review 1990;19:499-507.

18. Taylor DC, Bassett III FH. Syndesmosis ankle sprains: diagnosing the injury and aiding recovery. Physician Sportsmed 1993;21:39-46.

19. Taylor DC, Englehardt DL, Bassett FH.. Syndesmosis sprains of the ankle: the influence of heterotopic ossification. Am J Sports Med 1992;20:146-50.

20. Abraira VE, Ginty DD. The sensory neurons of touch. Neuron 2013;79:618-39.

21. Saladin K. Anatomy and physiology: The unity of form and function. Ohio: McGraw-Hill; 2010.

22. Adams R, Victor M, Ropper A. Principles of Neurology. 6th. Edition Singapore, McGraw-Hill Book; 1997.

23. Skraba J, Greenwald A. The role of the interosseous membrane on tibiofibular weightbearing. Foot Ankle Int 1984;4:301-4.

24. Rein S, Hanisch U, Zwipp H, et al. Comparative analysis of inter-and intraligamentous distribution of sensory nerve endings in ankle ligaments: a cadaver study. Foot Ankle Int 2013;34:1017-24.

25. Yeo ED, Kim HJ, Ahn J-H, et al. Can Bassett's ligament be removed? Knee Surg Sport $\operatorname{Tr} A$ 2016;24:1236-42.

Submission: June, 11, 2019

Acceptance: July 9, 2019 